\title{
NONEXISTENCE OF PERIODIC SOLUTIONS FOR THE FITZHUGH NERVE SYSTEM
}

\author{
BY \\ JITSURO SUGIE \\ Okayama University, Okayama, Japan \\ Dedicated to Professor Takasi Nagahara on his 60th birthday
}

1. Introduction. The system of differential equations developed by Hodgkin and Huxley is well known as a valid biological model of the squid giant axon membrane. However, since this system consists of four variables, the mathematical analysis for the Hodgkin-Huxley model is rather complicated. In [2,3] FitzHugh simplified this model and proposed the reduced system

$$
\begin{aligned}
& x^{\prime}=y-\frac{1}{3} x^{3}+x+I, \\
& y^{\prime}=\rho(a-x-b y),
\end{aligned}
$$

where the variable $x$ is the potential difference through the axon membrane, $y$ is the sodium inactivation (potassium activation), and the quantity $I$ is the current through the membrane. FitzHugh investigated the system (1.1) for special values of $I$ using numerical methods and phase space analysis. Several authors [4-6, 9] applied Hopf theory to (1.1), and studied the direction of bifurcation and stability of bifurcating periodic solutions of (1.1).

The purpose of this paper is to give a new criterion under which the system (1.1) and a system equivalent to (1.1) have no nonconstant periodic solutions.

Throughout this paper we assume that the parameters in (1.1) satisfy the conditions

$$
a \in \mathbf{R}, \quad 0<b<1, \quad \text { and } \rho>0 .
$$

For each $I \in \mathbf{R}$, the system (1.1) has the only critical point $\left(x_{I}, y_{I}\right)$ satisfying

$$
\begin{aligned}
x_{I}= & \sqrt[3]{\left\{3(I+a / b)+\sqrt{9(I+a / b)^{2}+4(1 / b-1)^{3}}\right\} / 2} \\
& +\sqrt[3]{\left\{3(I+a / b)-\sqrt{9(I+a / b)^{2}+4(1 / b-1)^{3}}\right\} / 2}
\end{aligned}
$$

and

$$
y_{I}=\left(a-x_{I}\right) / b .
$$

A simple argument shows that if $\rho b \geq 1$, then there exist no periodic solutions except for the critical point $\left(x_{I}, y_{I}\right)$. (For details, see Remark 3.2.) We therefore 
intend to discuss the case

$$
0<\rho b<1 .
$$

Let us take $\eta=x_{I}$ and $\eta_{0}=\sqrt{1-\rho b}$ as new parameters. Then we notice that $\eta_{0}^{2}<1$. Suppose that $\eta_{0}^{2}>\eta^{2}$; then all solutions of (1.1) are bounded and the critical point $\left(x_{I}, y_{I}\right)$ is unstable. Hence, by the Poincaré-Bendixson theorem (see, for example, [1]), there exists at least one nonconstant periodic solution of (1.1). Thus, it is quite reasonable to assume that

$$
\eta_{0}^{2} \leq \eta^{2}
$$

in Theorem $\mathrm{A}$ and Theorem 1.1 below.

Kaumann and Staude [7] gave the following result on the nonexistence of periodic solutions of (1.1).

TheOREM A [7]. Suppose that the assumptions (1.2) and (1.3) are satisfied. Further, suppose that either

$$
\eta_{0}^{2} \leq \eta^{2} \quad \text { and } \quad \eta_{0}^{2} \leq \frac{1}{b}-1
$$

or

$$
2 \eta_{0}^{2}-\left(\frac{1}{b}-1\right)<\eta^{2} \text { and } \frac{1}{b}-1<\eta_{0}^{2}
$$

Then the system (1.1) has no nonconstant periodic solutions.

If $\frac{1}{2}<b<1$, then the region given by (1.5) or (1.6) corresponds to the shaded portion in Fig. 1. This and succeeding figures were drawn with an $X-Y$ plotter by a computer.

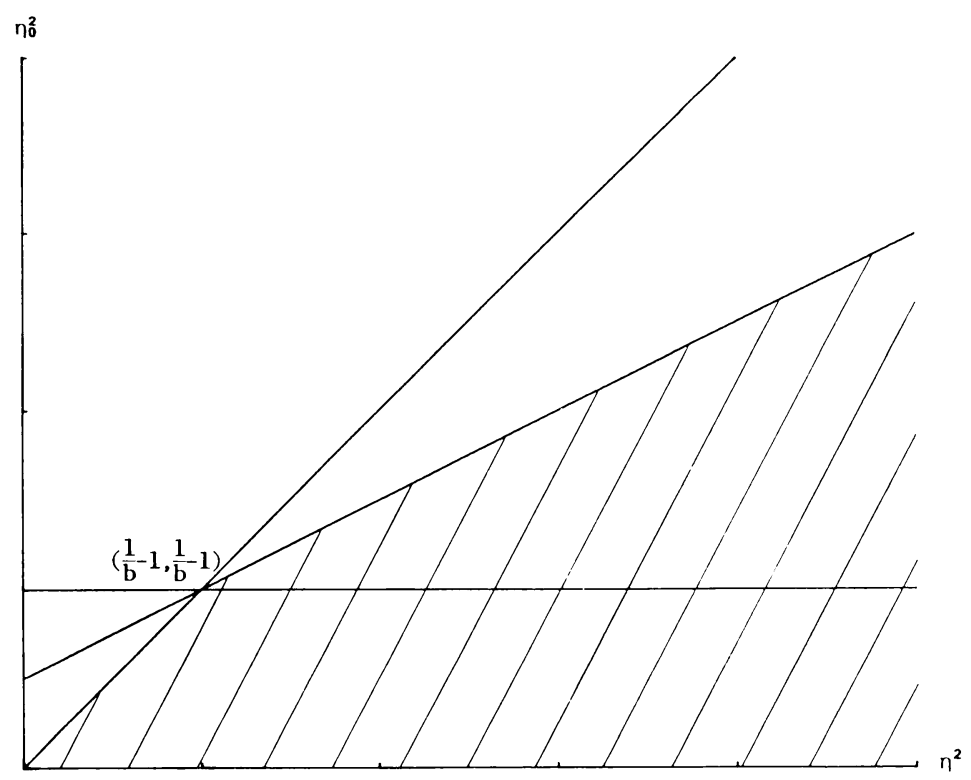

FIG. 1 
In the case $0<b \leq \frac{1}{2},(1.6)$ does not hold and (1.5) coincides with (1.4), and hence Theorem A shows that (1.4) implies there exist no nonconstant periodic solutions of (1.1). Thus, (1.4) is a necessary and sufficient condition for the nonexistence of periodic solutions of (1.1). However, in the case $\frac{1}{2}<b<1,(1.5)$ or (1.6) is not always necessary as Examples 4.1 and 4.3 in Sec. 4 show. It would be natural to conjecture that (1.4) implies there exist no nonconstant periodic solutions of (1.1) even if $\frac{1}{2}<b<1$. We indeed show that Theorem $\mathrm{A}$ is extended as follows.

TheOREM 1.1. Suppose that the assumptions (1.2) and (1.3) are satisfied. Further, suppose that either

$$
\begin{gathered}
\eta_{0}^{2} \leq \eta^{2} \\
\eta^{4}-4 \eta^{2} \eta_{0}^{2}+\eta_{0}^{4}+2\left(\frac{1}{b}-1\right) \eta^{2}-4\left(\frac{1}{b}-1\right) \eta_{0}^{2}+4\left(\frac{1}{b}-1\right)^{2} \geq 0 ;
\end{gathered}
$$

or

$$
2\left\{\eta_{0}^{2}+\left(\frac{1}{b}-1\right)\right\}^{3}<\eta^{2}\left\{\eta^{2}+3\left(\frac{1}{b}-1\right)\right\}^{2} .
$$

Then the system (1.1) has no nonconstant periodic solutions.

The region (1.7) or (1.8) is shown by the shaded portion in Fig. 2. In Example 4.2 we also show that the above conjecture is false.

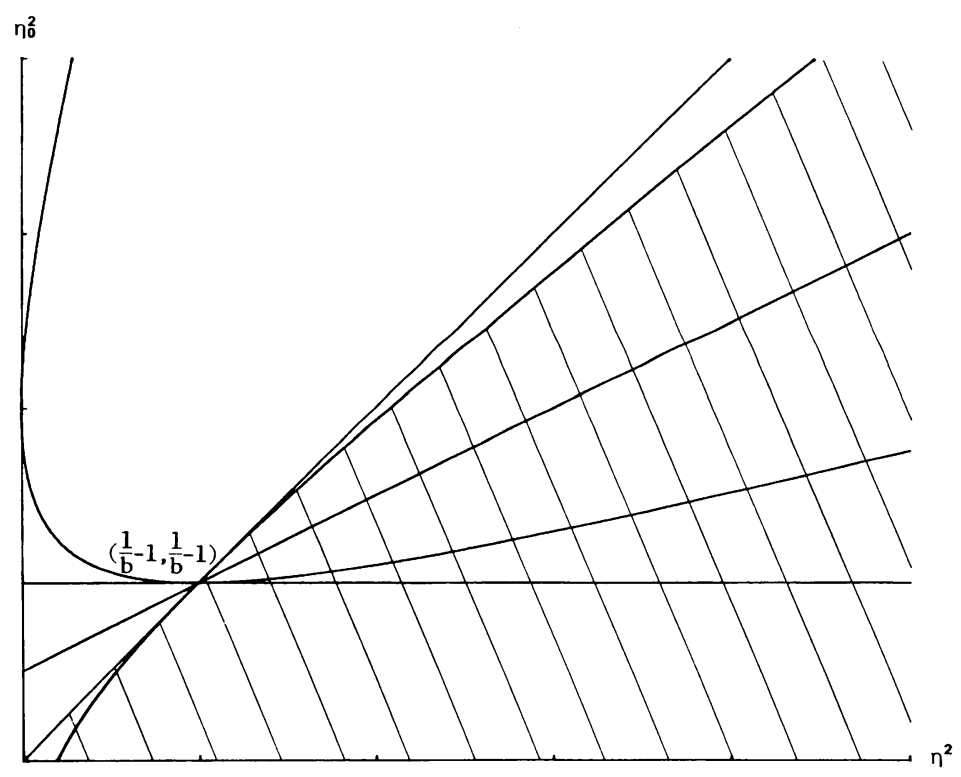

Fig. 2

2. Preliminary. Let us consider the Liénard system

$$
x^{\prime}=y-F(x), \quad y^{\prime}=-g(x)
$$


in which $F(x)$ and $g(x)$ are continuous functions on $\mathbf{R}$ satisfying locally a Lipschitz condition. We assume that

$$
F(0)=0 \text { and } \quad x g(x)>0 \text { if } x \neq 0 .
$$

Then the origin is the only critical point. Let $M=\min \left\{\int_{0}^{\infty} g(x) d x, \int_{0}^{-\infty} g(x) d x\right\}$ $(M$ may be $\infty$ ) and let

$$
w=G(x)=\int_{0}^{x}|g(\xi)| d \xi .
$$

Then by (2.2), $G(x)$ is strictly increasing. We denote the inverse function of $G(x)$ by $G^{-1}(w)$.

In a recent article [8], the author and Hara gave the following condition on $F(x)$ and $g(x)$ under which the system $(2.1)$ has no periodic solutions except for the origin.

THEOREM B [8]. Suppose that

$$
F\left(G^{-1}(-w)\right) \neq F\left(G^{-1}(w)\right) \text { for } 0<w<M .
$$

Then the system (2.1) has no nonconstant periodic solutions.

Now we turn to the FitzHugh nerve system (1.1). It is known that this system can be rewritten as

$$
\begin{aligned}
& x^{\prime}=y-\left\{\frac{1}{3} x^{3}+\eta x^{2}+\left(\eta^{2}+\rho b-1\right) x\right\}, \\
& y^{\prime}=-\rho b\left\{\frac{1}{3} x^{3}+\eta x^{2}+\left(\eta^{2}+\frac{1}{b}-1\right) x\right\},
\end{aligned}
$$

where $\eta$ is a new parameter (see $[4,7])$. Let

$$
F(x, \eta)=\frac{1}{3} x^{3}+\eta x^{2}+\left(\eta^{2}+\rho b-1\right) x
$$

and

$$
g(x, \eta)=\rho b\left\{\frac{1}{3} x^{3}+\eta x^{2}+\left(\eta^{2}+\frac{1}{b}-1\right) x\right\} .
$$

Then (2.2) is satisfied for all $\eta \in \mathbf{R}$. Thus, we can regard the system (2.4) as a special case of (2.1) for each fixed $\eta \in \mathbf{R}$ with $M=\infty$. By applying Theorem B, we wish to prove Theorem 1.1. Unfortunately, however, it is difficult to construct explicitly the inverse function $G^{-1}(w, \eta)$, and hence we must contrive to verify (2.3).

In the next section we will give some conditions which are equivalent to (2.3), and show that they are satisfied if (1.7) or (1.8) holds.

3. Proof of Theorem 1.1. Let $\eta_{0}=\sqrt{1-\rho b}$ as before. We first note that not only (1.7) but also (1.8) yields $\eta_{0}^{2} \leq \eta^{2}$. Therefore, for the sake of convenience, we divide the proof into the following two cases: (i) $4 \eta_{0}^{2}<\eta^{2}$; (ii) $\eta_{0}^{2} \leq \eta^{2} \leq 4 \eta_{0}^{2}$.

Suppose that $4 \eta_{0}^{2}<\eta^{2}$. Then we can verify

$$
x F(x, \eta)>0 \text { if } x \neq 0 .
$$

Since $G^{-1}(w, \eta)$ is strictly increasing for all $w \in \mathbf{R}$,

$$
G^{-1}(-w, \eta)<G^{-1}(0, \eta)=0<G^{-1}(w, \eta) \text { for } w>0 .
$$


Hence, we have

$$
F\left(G^{-1}(-w, \eta)\right)<0<F\left(G^{-1}(w, \eta)\right) \text { for } w>0,
$$

and therefore (2.3) is verified. Thus by Theorem B, the system (2.4) and its equivalent system (1.1) have no nonconstant periodic solutions.

Suppose that $\eta_{0}^{2} \leq \eta^{2} \leq 4 \eta_{0}^{2}$. By the transformations $x \rightarrow-x$ and $y \rightarrow-y$, the system (2.4) becomes

$$
\begin{aligned}
& x^{\prime}=y-\left\{\frac{1}{3} x^{3}-\eta x^{2}+\left(\eta^{2}-\eta_{0}^{2}\right) x\right\} \\
& y^{\prime}=-\rho b\left\{\frac{1}{3} x^{3}-\eta x^{2}+\left(\eta^{2}+\frac{1}{b}-1\right) x\right\} .
\end{aligned}
$$

Thus, we may only consider the case

$$
-2 \eta_{0} \leq \eta \leq-\eta_{0}
$$

In this case the function $F(x, \eta)$ has two real zeros besides $x=0$. Let $\beta$ and $\gamma$ be the zeros satisfying $\beta \leq \gamma$. Then

$$
\begin{aligned}
& \beta=\frac{1}{2}\left\{-3 \eta-\sqrt{3\left(4 \eta_{0}^{2}-\eta^{2}\right)}\right\} \geq 0, \\
& \gamma=\frac{1}{2}\left\{-3 \eta+\sqrt{3\left(4 \eta_{0}^{2}-\eta^{2}\right)}\right\}>0 .
\end{aligned}
$$

Define $\lambda^{*}=\frac{1}{3}\left(\eta_{0}-\eta\right)^{2}\left(2 \eta_{0}+\eta\right)$ and denote by $\alpha(\lambda), \beta(\lambda)$, and $\gamma(\lambda)$ the roots of the equation $F(x, \eta)=-\lambda$ for $0<\lambda \leq \lambda^{*}$ (refer to Fig. 3). Then we can see that

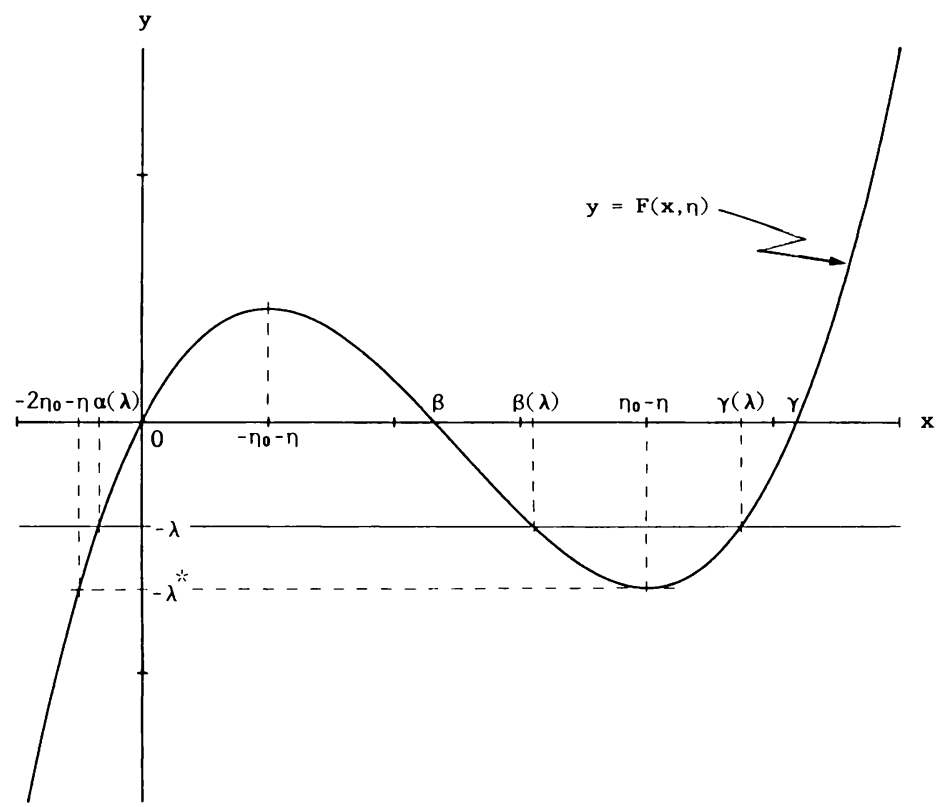

FIG. 3 


$$
\alpha(\lambda)<0<\beta(\lambda) \leq \gamma(\lambda)
$$

and

$$
\alpha(\lambda) \rightarrow 0, \quad \beta(\lambda) \rightarrow \beta, \quad \gamma(\lambda) \rightarrow \gamma \quad \text { as } \lambda \rightarrow 0 .
$$

As it is serious to verify (2.3) directly, we give some conditions equivalent to (2.3). For clarity of arguments, we show Claims 1 and 2 below.

Claim 1. Condition (2.3) is equivalent to

$$
\begin{array}{ll}
G(\alpha(\lambda), \eta)+G(\beta(\lambda), \eta) \neq 0 & \text { for } 0<\lambda \leq \lambda^{*}, \\
G(\alpha(\lambda), \eta)+G(\gamma(\lambda), \eta) \neq 0 & \text { for } 0<\lambda \leq \lambda^{*} .
\end{array}
$$

In fact, by the definitions of $\alpha(\lambda), \beta(\lambda)$, and $\gamma(\lambda)$, there exists $w_{0}>0$ such that

$$
F\left(G^{-1}\left(-w_{0}, \eta\right)\right)=F\left(G^{-1}\left(w_{0}, \eta\right)\right)
$$

if and only if there exists $\lambda_{0} \in\left(0, \lambda^{*}\right]$ such that

$$
G^{-1}\left(-w_{0}, \eta\right)=\alpha\left(\lambda_{0}\right) \text { and } G^{-1}\left(w_{0}, \eta\right)=\beta\left(\lambda_{0}\right)
$$

or

$$
G^{-1}\left(-w_{0}, \eta\right)=\alpha\left(\lambda_{0}\right) \text { and } G^{-1}\left(w_{0}, \eta\right)=\gamma\left(\lambda_{0}\right)
$$

that is,

$$
-G\left(\alpha\left(\lambda_{0}\right), \eta\right)=w_{0}=G\left(\beta\left(\lambda_{0}\right), \eta\right)
$$

or

$$
-G\left(\alpha\left(\lambda_{0}\right), \eta\right)=w_{0}=G\left(\gamma\left(\lambda_{0}\right), \eta\right) .
$$

Thus the claim is proved.

Let us calculate $G(\alpha(\lambda), \eta), G(\beta(\lambda), \eta)$, and $G(\gamma(\lambda), \eta)$. It follows from (2.5) and (2.6) that

$$
\begin{aligned}
G(x, \eta) & =\int_{0}^{x}|g(\xi, \eta)| d \xi \\
& = \begin{cases}\frac{\rho b}{12}\left\{x^{4}+4 \eta x^{3}+6\left(\eta^{2}+\frac{1}{b}-1\right) x^{2}\right\} & \text { for } x \geq 0 \\
-\frac{\rho b}{12}\left\{x^{4}+4 \eta x^{3}+6\left(\eta^{2}+\frac{1}{b}-1\right) x^{2}\right\} & \text { for } x<0\end{cases}
\end{aligned}
$$

and

$$
F(\alpha(\lambda), \eta)+\lambda=\frac{1}{3} \alpha(\lambda)^{3}+\eta \alpha(\lambda)^{2}+\left(\eta^{2}-\eta_{0}^{2}\right) \alpha(\lambda)+\lambda=0 .
$$

Hence, by (3.3),

$$
\begin{aligned}
-\frac{12}{\rho b} G(\alpha(\lambda), \eta) & =\alpha(\lambda)^{4}+4 \eta \alpha(\lambda)^{3}+6\left(\eta^{2}+\frac{1}{b}-1\right) \alpha(\lambda)^{2} \\
& =\{\alpha(\lambda)+\eta\}\left\{\alpha(\lambda)^{3}+3 \eta \alpha(\lambda)^{2}\right\}+3\left\{\eta^{2}+2\left(\frac{1}{b}-1\right)\right\} \alpha(\lambda)^{2} \\
& =3\left\{\eta_{0}^{2}+2\left(\frac{1}{b}-1\right)\right\} \alpha(\lambda)^{2}-3\left\{\eta\left(\eta^{2}-\eta_{0}^{2}\right)+\lambda\right\} \alpha(\lambda)-3 \eta \lambda .
\end{aligned}
$$


Similarly, we have

$$
\frac{12}{\rho b} G(\beta(\lambda), \eta)=3\left\{\eta_{0}^{2}+2\left(\frac{1}{b}-1\right)\right\} \beta(\lambda)^{2}-3\left\{\eta\left(\eta^{2}-\eta_{0}^{2}\right)+\lambda\right\} \beta(\lambda)-3 \eta \lambda
$$

and

$$
\frac{12}{\rho b} G(\gamma(\lambda), \eta)=3\left\{\eta_{0}^{2}+2\left(\frac{1}{b}-1\right)\right\} \gamma(\lambda)^{2}-3\left\{\eta\left(\eta^{2}-\eta_{0}^{2}\right)+\lambda\right\} \gamma(\lambda)-3 \eta \lambda .
$$

Thus, (3.5) becomes

$$
\left\{\eta_{0}^{2}+2\left(\frac{1}{b}-1\right)\right\}\{\alpha(\lambda)+\beta(\lambda)\} \neq \eta\left(\eta^{2}-\eta_{0}^{2}\right)+\lambda \text { for } 0<\lambda \leq \lambda^{*}
$$

and

$$
\left\{\eta_{0}^{2}+2\left(\frac{1}{b}-1\right)\right\}\{\alpha(\lambda)+\gamma(\lambda)\} \neq \eta\left(\eta^{2}-\eta_{0}^{2}\right)+\lambda \text { for } 0<\lambda \leq \lambda^{*} .
$$

Claim 2. Conditions (3.6) and (3.7) are equivalent to

$$
\left\{\eta_{0}^{2}+2\left(\frac{1}{b}-1\right)\right\}\{\alpha(\lambda)+\beta(\lambda)\}>\eta\left(\eta^{2}-\eta_{0}^{2}\right)+\lambda \text { for } 0<\lambda \leq \lambda^{*} .
$$

From (3.1), (3.2), and (3.4), we have

$$
\left\{\eta_{0}^{2}+2\left(\frac{1}{b}-1\right)\right\}\{\alpha(\lambda)+\gamma(\lambda)\} \rightarrow\left\{\eta_{0}^{2}+2\left(\frac{1}{b}-1\right)\right\} \gamma>0
$$

and

$$
\eta\left(\eta^{2}-\eta_{0}^{2}\right)+\lambda \rightarrow \eta\left(\eta^{2}-\eta_{0}^{2}\right) \leq 0
$$

as $\lambda \rightarrow 0$. Therefore, (3.7) implies

$$
\left\{\eta_{0}^{2}+2\left(\frac{1}{b}-1\right)\right\}\{\alpha(\lambda)+\gamma(\lambda)\}>\eta\left(\eta^{2}-\eta_{0}^{2}\right)+\lambda \text { for } 0<\lambda \leq \lambda^{*} .
$$

Since $\beta\left(\lambda^{*}\right)=\gamma\left(\lambda^{*}\right)$,

$$
\begin{aligned}
\left\{\eta_{0}^{2}+2\left(\frac{1}{b}-1\right)\right\}\left\{\alpha\left(\lambda^{*}\right)+\beta\left(\lambda^{*}\right)\right\} & =\left\{\eta_{0}^{2}+2\left(\frac{1}{b}-1\right)\right\}\left\{\alpha\left(\lambda^{*}\right)+\gamma\left(\lambda^{*}\right)\right\} \\
& >\eta\left(\eta^{2}-\eta_{0}^{2}\right)+\lambda^{*}
\end{aligned}
$$

This shows that (3.6) implies (3.8). On the other hand, it follows from (3.3) that

$$
\left\{\eta_{0}^{2}+2\left(\frac{1}{b}-1\right)\right\}\{\alpha(\lambda)+\beta(\lambda)\} \leq\left\{\eta_{0}^{2}+2\left(\frac{1}{b}-1\right)\right\}\{\alpha(\lambda)+\gamma(\lambda)\}
$$

for $0<\lambda \leq \lambda^{*}$. Therefore, (3.8) implies (3.6) and (3.7). Thus, the claim is proved.

Now we introduce a new parameter $\xi$ instead of $\lambda$. Consider the equation $F(x, \eta)=F(\xi, \eta)$ for $\eta_{0}-\eta \leq \xi<\gamma$. Then it is obvious that $\xi$ is a root of this equation. Let $\alpha(\xi)$ and $\beta(\xi)$ be other roots (see Fig. 4). Then by (2.5), we have

$$
\begin{aligned}
F(x, \eta)-F(\xi, \eta) & =\frac{1}{3} x^{3}+\eta x^{2}+\left(\eta^{2}-\eta_{0}^{2}\right) x-\frac{1}{3} \xi^{3}-\eta \xi^{2}-\left(\eta^{2}-\eta_{0}^{2}\right) \xi \\
& =\frac{1}{3}(x-\xi)\left\{x^{2}+(3 \eta+\xi) x+\left(3 \eta^{2}-3 \eta_{0}^{2}+3 \eta \xi+\xi^{2}\right)\right\} \\
& =\frac{1}{3}(x-\xi)\{x-\alpha(\xi)\}\{x-\beta(\xi)\}
\end{aligned}
$$




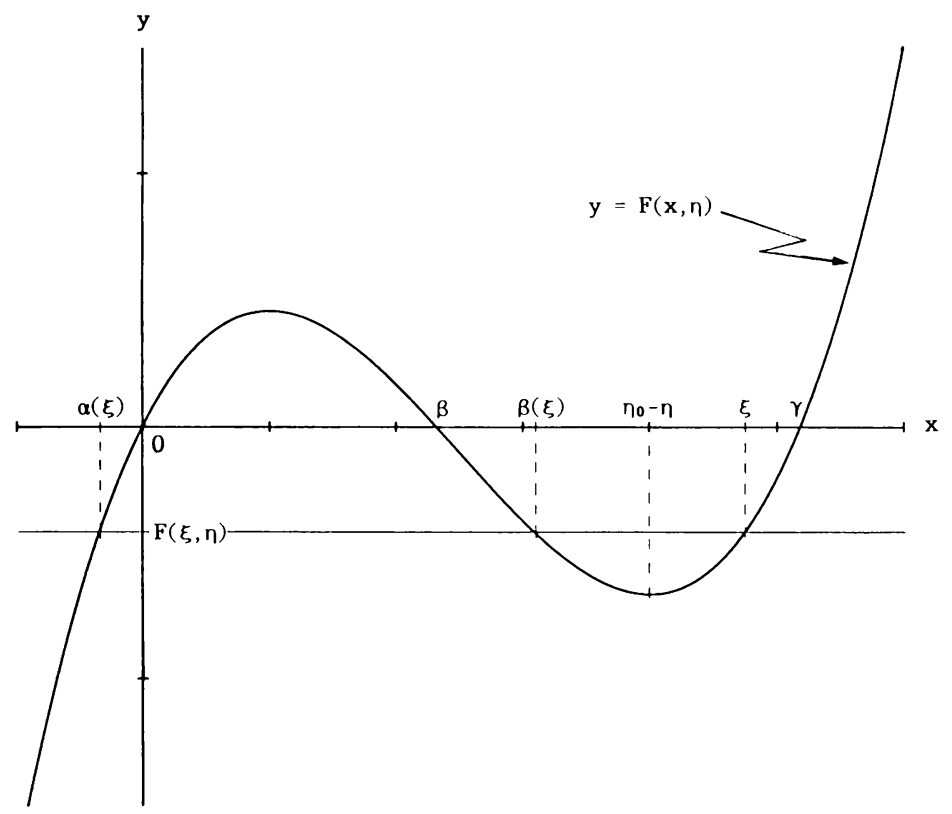

FIG. 4

and hence

$$
\alpha(\xi)+\beta(\xi)=-(3 \eta+\xi) .
$$

Thus, we can rewrite (3.8) as

$$
-\left\{\eta_{0}^{2}+2\left(\frac{1}{b}-1\right)\right\}(3 \eta+\xi)>\eta\left(\eta^{2}-\eta_{0}^{2}\right)-F(\xi, \eta)
$$

for $\eta_{0}-\eta \leq \xi<\gamma$. For the sake of simplicity, we define

$$
\begin{aligned}
H(\xi, \eta)= & F(\xi, \eta)-\left\{\eta_{0}^{2}+2\left(\frac{1}{b}-1\right)\right\}(3 \eta+\xi)-\eta\left(\eta^{2}-\eta_{0}^{2}\right) \\
= & \frac{1}{3} \xi^{3}+\eta \xi^{2}+\left\{\eta^{2}-2 \eta_{0}^{2}-2\left(\frac{1}{b}-1\right)\right\} \xi-3 \eta\left\{\eta_{0}^{2}+2\left(\frac{1}{b}-1\right)\right\} \\
& -\eta\left(\eta^{2}-\eta_{0}^{2}\right) .
\end{aligned}
$$

Then this inequality becomes

$$
H(\xi, \eta)>0 \text { for } \eta_{0}-\eta \leq \xi<\gamma .
$$

By the above arguments, it turns out that (2.3) is equivalent to (3.9) and so if (3.9) is satisfied, then by Theorem B the systems (2.4) and (1.1) have no nonperiodic solutions. In the following, we will show that (1.7) or (1.8) implies (3.9).

It is clear that $H(\xi, \eta)$ is monotone decreasing for $-\eta-\sqrt{2\left\{\eta_{0}^{2}+(1 / b-1)\right\}} \leq \xi \leq$ $-\eta+\sqrt{2\left\{\eta_{0}^{2}+(1 / b-1)\right\}}$, and monotone increasing for $\xi<-\eta-\sqrt{2\left\{\eta_{0}^{2}+(1 / b-1)\right\}}$ or $\xi>-\eta+\sqrt{2\left\{\eta_{0}^{2}+(1 / b-1)\right\}}$. By (3.2), we have

$$
\beta=-(3 \eta+\gamma),
$$


and hence by (3.1) and (3.2),

$$
\begin{aligned}
H(\gamma, \eta) & =F(\gamma, \eta)-\left\{\eta_{0}^{2}+2\left(\frac{1}{b}-1\right)\right\}(3 \eta+\gamma)-\eta\left(\eta^{2}-\eta_{0}^{2}\right) \\
& =\left\{\eta_{0}^{2}+2\left(\frac{1}{b}-1\right)\right\} \beta-\eta\left(\eta^{2}-\eta_{0}^{2}\right) \geq 0 .
\end{aligned}
$$

First estimation. Suppose that (1.7) holds. Then an easy calculation shows that

$$
\gamma \leq-\eta+\sqrt{2\left\{\eta_{0}^{2}+(1 / b-1)\right\}} .
$$

Therefore, since $-\eta-\sqrt{2\left\{\eta_{0}^{2}+(1 / b-1)\right\}}<\eta_{0}-\eta, H(\xi, \eta)$ is monotone decreasing for $\eta_{0}-\eta \leq \xi \leq \gamma$, and hence by (3.10),

$$
H(\xi, \eta)>H(\gamma, \eta) \geq 0 \text { for } \eta_{0}-\eta \leq \xi<\gamma .
$$

Second estimation. Suppose that (1.8) holds, then

$$
-\eta^{3}-3\left(\frac{1}{b}-1\right) \eta>\left\{\eta_{0}^{2}+\left(\frac{1}{b}-1\right)\right\} \sqrt{2\left\{\eta_{0}^{2}+(1 / b-1)\right\}}
$$

Therefore, we have

$$
\begin{aligned}
H(\xi, \eta) & \geq H\left(-\eta+\sqrt{2\left\{\eta_{0}^{2}+(1 / b-1)\right\}}, \eta\right) \\
& =-\frac{4}{3} \eta^{3}-4\left(\frac{1}{b}-1\right) \eta-\frac{4}{3}\left\{\eta_{0}^{2}+\left(\frac{1}{b}-1\right)\right\} \sqrt{2\left\{\eta_{0}^{2}+(1 / b-1)\right\}}>0
\end{aligned}
$$

for $\eta_{0}-\eta \leq \xi<\gamma$.

Hence, in either case, (3.9) is verified. Thus the proof of Theorem 1.1 is now complete.

REMARK 3.1. By a simple argument, we can also see that if neither (1.7) nor (1.8) is satisfied, then there exists $\xi_{0} \in\left[\eta_{0}-\eta, \gamma\right)$ such that $H\left(\xi_{0}, \eta\right) \leq 0$. Hence, the following equivalence relation holds in the case $\eta_{0}^{2} \leq \eta^{2} \leq 4 \eta_{0}^{2}$ :

$$
\begin{aligned}
(2.3) & \rightleftarrows(3.5) \rightleftarrows((3.6) \text { and }(3.7)) \\
& \rightleftarrows(3.8) \rightleftarrows(3.9) \rightleftarrows((1.7) \text { or }(1.8)) .
\end{aligned}
$$

REMARK 3.2. If $\rho b \geq 1$, then by (2.5) it is easily seen that $F(x, \eta)$ is monotone increasing for all $x \in \mathbf{R}$. Thus, the same argument of the case $4 \eta_{0}^{2}<\eta^{2}$ shows that (2.3) is satisfied, and so the system (1.1) has no nonconstant periodic solutions. (To be precise, the critical point is a globally asymptotically stable solution of (1.1).)

4. Numerical examples. In this section we present the phase portraits of some concrete systems (Figs. 5-7) to show how we should rank Theorem 1.1. The first example does not satisfy the conditions in Theorem A, but satisfies those in Theorem 1.1 .

EXAMPle 4.1. Consider the system (2.4) where the parameters satisfy

$$
b=\frac{4}{5}, \quad \rho=\frac{5}{16}, \quad \text { and } \quad \eta=-1 .
$$




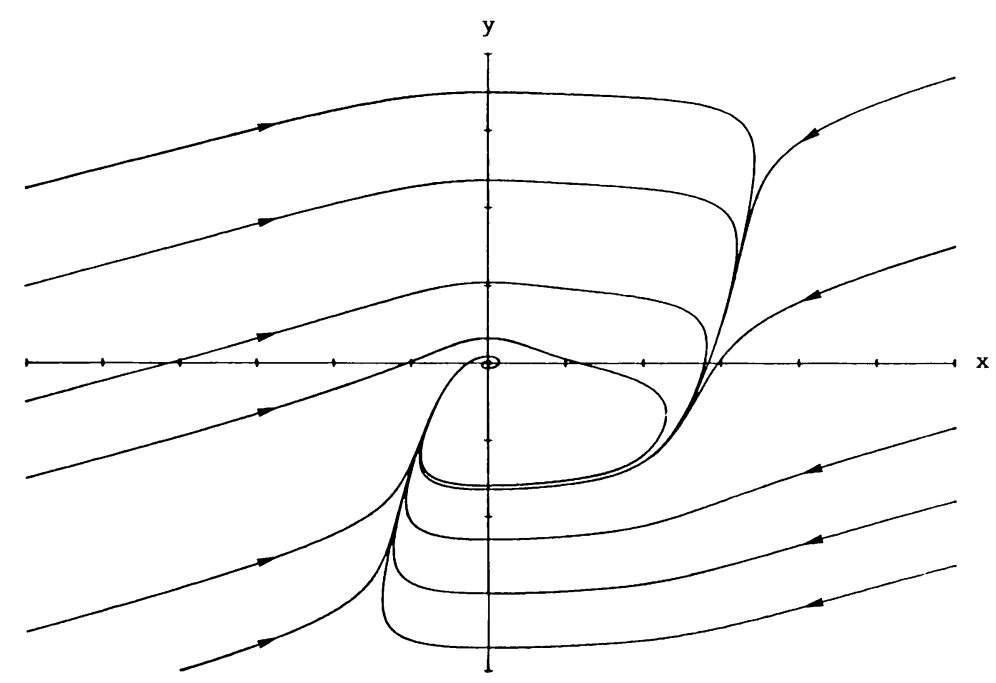

FIG. 5

By numerical methods, we can see that all solutions circle clockwise around the origin and tend toward it as $t \rightarrow \infty$, and hence the system (2.4) has no periodic solutions except for the origin (see Fig. 5).

In this example, the assumptions (1.2) and (1.3) are satisfied. However, since

$$
\eta_{0}^{2}=1-\rho b=\frac{3}{4}>\frac{1}{4}=\frac{1}{b}-1
$$

and

$$
2 \eta_{0}^{2}-\left(\frac{1}{b}-1\right)=\frac{5}{4}>1=\eta^{2}
$$

neither (1.5) nor (1.6) is satisfied. Hence Theorem A cannot be applied to Example 4.1. On the other hand, we have

$$
2\left\{\eta_{0}^{2}+\left(\frac{1}{b}-1\right)\right\}^{3}=2<\frac{49}{16}=\eta^{2}\left\{\eta^{2}+3\left(\frac{1}{b}-1\right)\right\}^{2}
$$

and so (1.8) is satisfied. Thus by Theorem 1.1, the system (1.1) and its equivalent system (2.4) have no nonconstant periodic solutions.

From the reason mentioned in Sec. 1 it seems that

$$
\eta_{0}^{2} \leq \eta^{2}
$$

is a necessary and sufficient condition for the nonexistence periodic solutions of (2.4). But the following example shows that this conjecture is not true for $\frac{1}{2}<b<1$.

EXAMPLE 4.2. Consider the system (2.4) where the parameters satisfy

$$
b=\frac{9}{10}, \quad \rho=\frac{1}{4}, \quad \text { and } \quad \eta=-\frac{9}{10} .
$$

Then $\eta_{0}^{2}=1-\rho b=\frac{31}{40}<\frac{81}{100}=\eta^{2}$ and so $(4.1)$ is satisfied. On the other hand, we 
have

$$
\begin{aligned}
\eta^{4}- & 4 \eta^{2} \eta_{0}^{2}+\eta_{0}^{4}+2\left(\frac{1}{b}-1\right) \eta^{2}-4\left(\frac{1}{b}-1\right) \eta_{0}^{2}+4\left(\frac{1}{b}-1\right)^{2} \\
< & \eta^{2}\left(\eta^{2}-4 \eta_{0}^{2}\right)+1+2\left(\frac{1}{b}-1\right) \eta^{2}+4\left(\frac{1}{b}-1\right)^{2} \\
= & \frac{81}{100} \times\left(\frac{81}{100}-\frac{31}{10}\right)+1+\frac{9}{50}+\frac{4}{81}<0
\end{aligned}
$$

and

$$
\begin{aligned}
2\left\{\eta_{0}^{2}+\left(\frac{1}{b}-1\right)\right\}^{3} & =2 \times\left(\frac{31}{40}+\frac{1}{9}\right)^{3}>2 \times\left(\frac{5}{6}\right)^{3} \\
& =\frac{125}{108}>\frac{42849}{40000}=\frac{81}{100} \times\left(\frac{23}{20}\right)^{2} \\
& >\frac{81}{100} \times\left(\frac{81}{100}+\frac{1}{3}\right)^{2}=\eta^{2}\left\{\eta^{2}+3\left(\frac{1}{b}-1\right)\right\}^{2} .
\end{aligned}
$$

Hence neither (1.7) nor (1.8) is satisfied. As shown in Fig. 6, the system (2.4) has two periodic solutions except for the origin. (To be precise, a small unstable limit cycle and a large stable limit cycle.)

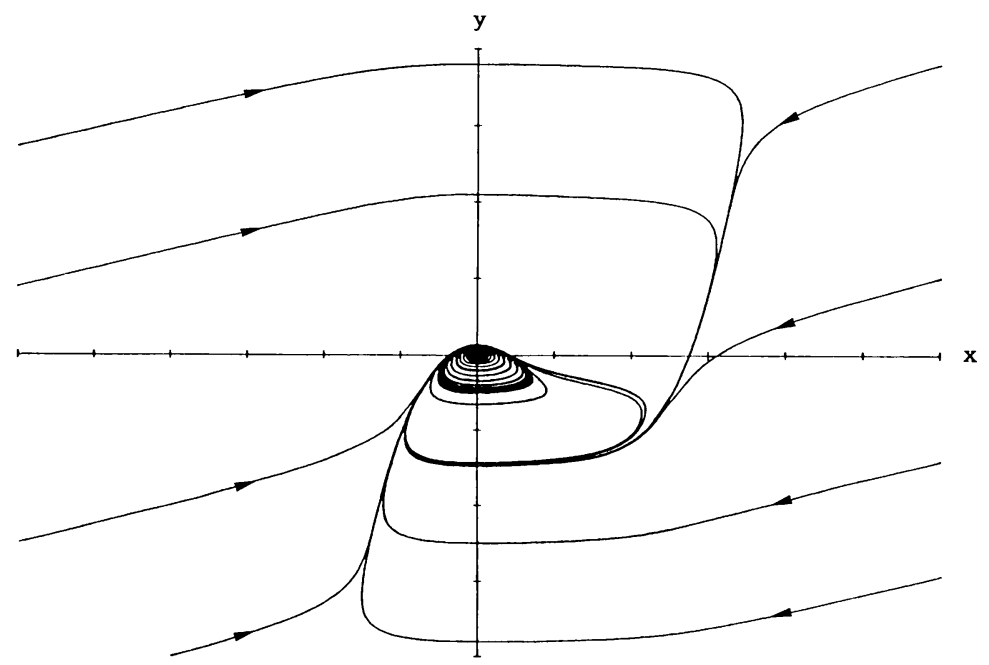

FIG. 6

Finally, we give an example to show that Theorem 1.1 is a sufficient condition for the nonexistence of periodic solutions of (2.4), but is not a necessary condition for this problem.

EXAMPLE 4.3. Consider the system (2.4) where the parameters satisfy

$$
b=\frac{5}{6}, \quad \rho=\frac{3}{10}, \quad \text { and } \quad \eta=-\frac{2}{\sqrt{5}} \text {. }
$$




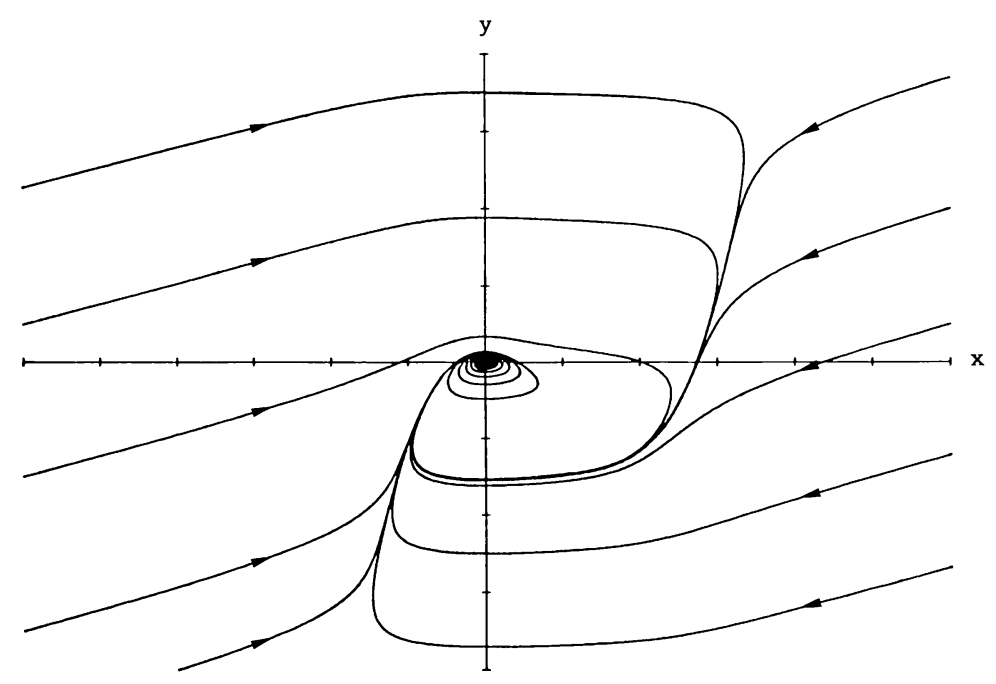

FIG. 7

Then, as in Example 4.2, we can verify that neither (1.7) nor (1.8) is satisfied, and so Theorem 1.1 cannot be applied to Example 4.3. Fig. 7 shows that the system (2.4) has no periodic solutions except for the origin.

Although Example 4.3 suggests that a better result than Theorem 1.1 exists, it would be difficult to achieve a satisfactory one.

Acknowledgment. This work was partially supported by Grant-in-Aid for Scientific Research 02854009.

\section{REFERENCES}

[1] E. A. Coddington and N. Levinson, Theory and Ordinary Differential Equations, McGraw-Hill, New York, 1955

[2] R. FitzHugh, Thresholds and plateaus in the Hodgkin-Huxley nerve equations, J. Gen. Phys. 43, 867-896 (1960)

[3] R. FitzHugh, Impulses and physiological states in theoretical models of nerve membrane, Biophys. J. 1, 445-466 (1961)

[4] K. P. Hadeler, U. an der Heiden, and K. Schumacher, Generation of the nervous impulse and periodic oscillations, Biol. Cybernet. 23, 211-218 (1976)

[5] I. D. Hs̈u and N. D. Kazarinoff, An applicable Hopf bifurcation formula and instability of small periodic solutions of Field-Noyes model, J. Math. Anal. Appl. 55, 61-89 (1976)

[6] I. D. Hs̈u, A higher-order Hopf bifurcation formula and its application to FitzHugh's nerve conduction equations, J. Math. Anal. Appl. 60, 47-57 (1977)

[7] E. Kaumann and U. Staude, Uniqueness and nonexistence of limit cycles for the FitzHugh equation, Equadiff 82 (H. W. Knobloch and K. Schmitt, eds.), Lecture Notes in Math., vol. 1017, SpringerVerlag, 1983, pp. 313-321

[8] J. Sugie and T. Hara, Non-existence of periodic solutions of the Lienard system, scheduled for J. Math. Anal. Appl. 159, No. 1 (1991)

[9] W. C. Troy, Bifurcation phenomena in FitzHugh's nerve conduction equations, J. Math. Anal. Appl. 54, 678-690 (1976) 